\title{
Developing smoking cessation program for older Canadian people: an application of Precede-Proceed model
}

\author{
Khaldoun M. Aldiabat ${ }^{1, ~}$, Carole-Lynne Le Navenec ${ }^{2}$ \\ ${ }^{1}$ School of Nursing, University of Northern British Columbia, 3333 University Way,Prince George, BC, V2N 4Z9, Canada \\ ${ }^{2}$ Faculty of Nursing, University of Calgary, 2500 University Drive N.W, Calgary, AB, T2N 1N4, Canada \\ Email address: \\ aldiabat@unbc.ca(K. M. Aldiabat), cllenave@ucalgary.ca(C. L. L. Navenec)
}

\section{To cite this article:}

Khaldoun M. Aldiabat, Carole-Lynne Le Navenec. Developing Smoking Cessation Program for Older Canadian People: An Application of Precede-Proceed Model. American Journal of Nursing Science Vol. 2, No. 3, 2013, pp. 33-39.

doi: 10.11648/j.ajns.20130203.13

\begin{abstract}
Precede-Proceed model is a flexible and appropriate to develop a smoking cessation/prevention program. The main purpose of this paper is to develop a preliminary hypothetical health promotion program that is focused on smoking cessation / prevention for older Canadian people who smoke and live in nursing homes to improve their quality of life. It is hoped that advanced-practice gerontology nurses in a range of health settings will be able to gain insights from this paper to help older Canadian people who smoke to quit tobacco consistently and effectively. However, because this model assumes that health behavior is a complex and multidimensional process, the advanced- practice gerontology nurses who will staff this kind of practice will be guided by this framework to use their educational and practical knowledge regarding health promotion and smoking cessation/prevention programs, critical thinking, communication skills, and decision-making abilities.
\end{abstract}

Keywords: Smoking Cessation, Smoking Prevention, Older People, Health Promotion, Gerontology Nurse, Advanced Practice Nurse, Precede-Proceed

\section{Introduction}

The prevalence and the negative influences of cigarettes smoking on the health of older people are well reported in the literature (British Columbia Ministry of Health, 2005, Edwards \& Mawani, 2006, Health Canada, 2002). The prevalence of cigarettes smoking among North American older people (65 and older) was 12\% (Kaplan, Newsom, \& McFarland, 2002). Whereas the overall smoking prevalence among Unites States older population was $10.2 \%$ (Doolan \& Froelicher, 2008), the smoking rate among older Canadians was 9\% (Public Health Agency of Canada, 2009). The highest smoking rate among North American older people has been found among Aboriginal Canadians aged 65 and older. According to Statistics Canada (2007), smoking rates among those older population were as the following: $22 \%$ among first nations, $22 \%$ among Métis, 36\% among Inuit. Although there is no new smoking prevalence reports of older Canadians could be found, the above are convincing statistics that the smoking rate among older Canadian people, especially Aboriginals is still high.

Smoking cigarettes among older people has a range of negative influences on their health and well-being. It has been reported in the previous literature that smoking among those population is classified as one of the highest risk factors that contributing to cause seven chronic diseases and increases the mortality rate among older people (Carosella, Ossip-Klein, Watt, \& Podgorski, 2002). Example of these chronic diseases includes: cardiovascular diseases, lungs; kidneys and pancreatic cancers, cerebrovascular accident, and different gum and teeth diseases (British Columbia Ministry of Health, 2005, Health Canada, 2002, US Department of Health and Human Services, 2004). However, the mortality rate among older people related to their current smoking behavior is twice than those who are in the same age but never smoked (Health Canada, 2002). In addition to the multidimensional negative influence of smoking, previous studies indicated that smoking interferes negatively with 
many medications commonly prescribed to the older people to make them over or less effective (Watt , Carosella, Podgorski, \& Ossip-Klein, 2004, Health Canada, 2002).

Having discussed briefly the smoking prevalence and related morbidity and mortality rates among older people, let us turn now to discuss what are the health benefits of smoking cessation among those populations? In contrast to the belief that it is too late for older people who smoke to quit, Husten et al. (1997) reported that "quitting smoking has a major and immediate benefits for smokers of all ages, and these benefits accrue to those who already have smoking- related diseases, as well as to those who do not. Smoking cessation at any age reduces the overall risk of death" (pp. 175-176). Therefore, the smoking cessation programs for older people aims not only to decrease the mortality and morbidity rates caused by smoking, but also to improve the quality of life for those people (Edwards \& Mawani, 2006, La Croix \& Omenn, 1992).

No studies or smoking cessation programs designed for older Canadian people who smoke at nursing homes could be found in the literature. Thus, the main purpose of this paper is to develop a preliminary hypothetical health promotion program that is focused on smoking cessation / prevention for older Canadian people who smoke and live in nursing homes to improve their quality of life. It is hoped that advanced-practice gerontology nurses in a range of health settings will be able to gain insights from this paper to help older Canadian people who smoke to quit tobacco consistently and effectively.

This program will be built on insights gleamed from the literature on health promotion, and organized in terms of the phases incorporated in the PRECEDE-PROCEED model (see figure 1) (Green \& Kreuter, 2005).

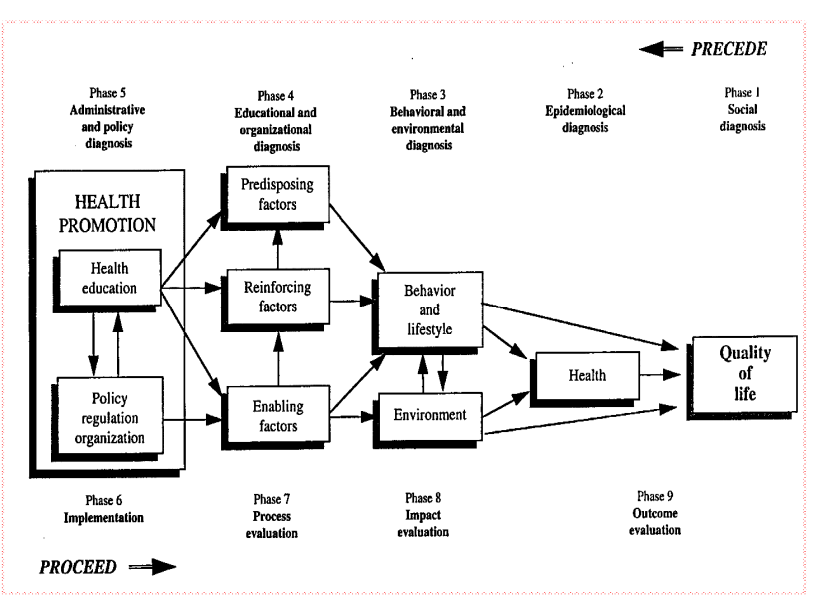

Figure 1. Generic representation of the PRECEED-PROCEED Model for Health Promotion, Planning and Evaluation (Green \& Kreuter, 2005). Retrieved from www.google.ca

\section{Precede-Proceed Model}

In this section, the discussion will address the following three questions: (1) what is the PRECEDE-PROCEED model? (2) Why has this model been considered as a possible to use for the development of a smoking cessation/ prevention program for older Canadian people who smoke and live in nursing homes? And, (3) how will this model be applicable for developing a health promotion program at the organizational (e.g., nursing homes and long-term facilities) level?

\subsection{Firs Question: What Is The PRECEDE-PROCEED Model?}

The Precede-Proceed model is a multi-theoretical based framework developed by Lawerence Green and Marshal Kreuter over 40 years ago. It is used to assess health related behaviors and environments that affect health and quality of life needs, and to design, implement and evaluate health promotion programs that improve quality of life for those who need these programs (Green \& Kreuter, 1991). In other words, it provides a comprehensive map for applying theories in evidence-based practice to plan and implement the most appropriate strategies that may solve health problems and improve quality of life (Gielen \& McDonald, 1997).

Precede stands for Predisposing, Reinforcing, and Enabling Constructs in Educational Diagnosis and Evaluation. This part of the model serves as a diagnostic planning process that assists in developing health promotion programs, because it assumes that educational diagnosis must be identified before an intervention, just as the formulation of nursing diagnoses should precede nursing planning and intervention (Gielen \& McDonald, 1997; Green \& Kreuter, 1991). In 1991, the original authors added Proceed, which stands for Policy, Regulatory, and Organizational Constructs in Educational and Environmental Development. This second part guides the implementation and evaluation of the programs designed using Proceed to develop an untraditional health promotion program that combined health education and the contexts (Green \& Kreuter, 2005).

The Precede-Proceed consists of nine phases (see Figure 1, Precede-Proceed model) based on the premise "that health behaviors are complex, multidimensional, and influenced by a variety of factors" (Gielen \& McDonald, 1997, p.360). Proceed involves five phases (phase one to phase five) whereas Proceed consists of four phases (phase six to phase nine). In the next two paragraphs, we will provide a brief description about each phase based on the work of Gielen and McDonald (1997) and Green and Kreuter (1991). A more detailed description and application of these phases will be discussed in the application section.

Consideration is given during phase one to determining the social problems or the quality of life needs of a given population. The task of phase two is to identify the health determinants of these problems and needs noted in faze one. In phase three, attention is given to identify the behavioral and environmental determinants of the health problems. 
Predisposing factors (including information, attitudes, beliefs, values, and perception about the desired behavior change), enabling factors (including the contextual factors that facilitate or hinder the desired behavioral and environmental changes), and reinforcing factors (rewards and evaluation of others that may encourage or discourage continuing the desired behavior change) are identified during phase four. Phase five, the last phase in PRECEDE, pertains to the assessment of organizational policy, administrative facilitations, intervention alignment, and limitations that discourage implementation of the health promotion program.

In phase six, the interventions that were identified in phase five are ready now for implementation. Phases seven, eight, and nine entail process, impact, and outcome evaluation respectively. These evaluation techniques have been designed to assess certain tasks during the health promotion program. For example, process evaluation assesses if the program has been implemented according to planned protocol. Impact evaluation determines the effect of the intervention on the change in the behavioral and environmental factors. The final one is the outcome evaluation, which is designed to evaluate the major effects of the interventions on the health and quality of life of the target population.

Having addressed the first question (i.e., what is the PRECEDE-PROCEED model?), let us now turn to answer the second one (Why has this model been considered as a possible to use for the development of a smoking cessation/ prevention program for older Canadian people who smoke and live in nursing homes?).

\subsection{Second Question: Why Has This Model Been Considered As A Possible To Use For The Development of A Smoking Cessation/Prevention Program For Older Canadian People Who Smoke And Live In Nursing Homes?}

The Precede-Proceed model has been chosen to develop this program for two major reasons. First, this model provides clear steps to plan health promotion programs for actual or potential health problems and through the three levels of prevention (primary, secondary, and tertiary) (Green \& Kreuter, 2005). For example, the Canadian Lung Association (CLA) has developed a national lung health framework to improve quality of life for those who have actual or potential respiratory health problem (CLA, 2008). Analysis of this framework shows clearly that it was developed based on the Precede-Proceed model because it starts from quality of life and social diagnosis and returns back to include the determinants of respiratory health status. Hence, maintenance of any health behavior change such as smoking among older Canadian people who smoke can be achieved by giving the same attention to both treatment and prevention.

Second, this model (precede-proceed) was built on numerous health and behavior theories such as health belief model, health promotion model, and social learning theory (Green \& Kreuter, 1991). Gielen and McDonald (1997) contended that the success and wide use of this model for developing health promotion programs is related to its strong theoretical base. Hence, it is expected that use of this successful theoretically-based model to develop a smoking cessation/prevention program for older Canadian people who smoke has many advantages. For example, using Precede-Proceed model not only contributes to the success of that program, but also convinces decision makers, stakeholders, fund supporters, and other partners regarding the benefits of building the health promotion program.

In summary, the above two factors provide strong indications for choosing the Precede-Proceed model to develop a smoking cessation/prevention program. However, because this model assumes that health behavior is a complex and multidimensional process, the advancedpractice gerontology nurse will also need multidimensional skills and knowledge from different sources to use this model for evidence-based health promotion practice (Green \& Kreuter, 2005). Hence, the advanced- practice gerontology nurses who will staff this kind of practice must use their educational and practical knowledge regarding health promotion and smoking cessation/prevention programs, critical thinking, communication skills, and decision-making abilities. Some specific examples of these skills and abilities will be discussed next.

\subsection{Third Question: How Will This Model Be Applicable for Developing a Health Promotion Program at the Organizational (e.g., Nursing Homes and Long-Term Facilities) Level?}

The advanced-practice gerontology nurses and other program partners have a responsibility to determine the mission, vision, objectives, and action plan of the health promotion program. The following hypothetical material pertains to the formulation of the following: mission statement, vision, hypothesis, goal, objectives, and action plan of a smoking cessation/prevention health promotion program, which will be designed by nurses for older Canadian people who smoke in nursing homes.

\subsubsection{The Vision}

"Safe and healthy nursing home environment"

\subsubsection{The Mission}

"To enhance health and quality of life for all persons in the nursing home through developing a smoking cessation and prevention program to be delivered by advancedpractice gerontology nurses for older Canadian people who smoke".

\subsubsection{Hypothesis:}

"If we succeed in implementing this program, then the number of older Canadian people who smoke will be reduced, their quality of life will be enhanced, the mortality 
and morbidity rates related to smoking will be decreased, the environment of nursing home settings will become safer and healthier, the health and quality of life of other employees and people who do not smoke will be protected from second-hand smoke, and the productivity of the organization will be increased by reducing other risk factors that can be caused by smoking".

\subsubsection{Goal}

"To cease and prevent smoking among older Canadian people who smoke and live in nursing homes".

\subsubsection{Objectives}

The following are only examples of the salient objectives

\subsubsection{1. (A) Outcome Objective}

"Within five years of initiating the program, self reporting by older Canadian people who smoke and live in nursing home settings will indicate that $50 \%$ have completely quit smoking, $25 \%$ think they will quit smoking next year, $25 \%$ no longer smoke inside their rooms but in designed smoking rooms inside the nursing home, $100 \%$ of those who have never smoked still continue as nonsmokers, and only $1 \%$ of those who have quit smoking relapsed".

\subsubsection{2. (b) Process objective}

"To prepare one small, private, equipped room inside the nursing home setting to be used as a clinic in which the advanced- practice gerontology nurses and related specialists provide counseling and other treatments that are culturally meaningful to the clients being served".

\section{The Action Plan for the Health Promotion Program}

In this section, we will discuss the action plan for this hypothetical smoking cessation and prevention program that is based on the nine phases of the PRECEDEPROCEED model (Green \& Kreuter, 2005; Gielen \& McDonald, 1997). Insights for this action plan have been partially derived from the National Lung Health Framework for Canada (CLA, 2008).

\subsection{Phase 1: Social Diagnosis: Assessing Quality of Life Concerns}

During this phase, it is expected that advanced-practice gerontology nurses determine the perception of older Canadian people who smoke regarding their own needs or quality of life through multiple information-gathering activities. Example of these activities include knowledge about the social needs of older Canadian people who smoke from their perspective, and how various contextual factors in nursing home may influence their health and quality of life. In addition, these needs have been collected through different data collection methods. For example, advanced-practice gerontology nurses assess the social needs by reviewing the literature, conducting individual sessions and/or focus groups, or through individual interviews, questionnaires and surveys, organizational files, social indicators, and organizational forums. Hence, to identify specific social diagnosis needs, advanced-practice gerontology nurses need to involve the older Canadians people in the nursing home who smoke, as well as other staff and people in nursing home because the issue of second-hand smoke and related concerns.

\subsection{Phase 2: Epidemiological Diagnosis}

Following the identification of the social needs of older Canadians people who smoke, as well as other staff and people in the nursing home, the second phase is concerned with assessing and identifying the epidemiological details of the smoking among older Canadians people who smoke in the nursing home and characteristics of those who are at risk related to smoking. For epidemiological diagnosis, advanced-practice gerontology nurses use surveys, data from the department records, and critical reviews of the literature for information regarding mortality and morbidity data as well as risk factors identified in the literature that assessed factors contributing to smoking among older Canadians people who smoke and live in the nursing home. Hence, knowing the epidemiological factors helps nurses to better address the health problem through strategies to control them.

\subsection{Phase 3: Behavioral and Environmental Diagnosis}

The aim of this phase is to determine the behavioral and environmental correlates of smoking among older Canadians people in the nursing home. Behavioral correlates are behaviors or lifestyles that contribute to their smoking pattern, whereas environmental correlates are social and physical factors that encourage those older persons to smoke. The delineation of these factors should first be accomplished with a thorough review of the literature related to the behavioral and environmental factors that contribute to smoking among older Canadians people in the nursing home. Literature review will help advanced practice gerontology nurses to identify health and environmental factors that contribute to the social needs through using a reductionist approach (i.e., from very general to specific factors). To illustrate, the advancedpractice gerontology nurses think about all behavioral and health factors that may influence smoking among older Canadians people who smoke in the nursing home in general, then choose the most factors that fit the situation.

The following critical questions are expected from the advance practice gerontology nurse to assess behavioral and environmental factors of smoking in the nursing home: (1) what factors have contributed to smoking among older Canadian people in this nursing home setting? (2)What factors have encouraged them to smoke within this nursing home setting? (3) Are there more barriers than facilitators 
to older Canadian people who smoke to stop smoking within this nursing home setting?

The advanced practice gerontology nurse may be able to use the results of a study to determine what are the salient health behavioral and environmental factors that contributed to smoking among those people (e.g., relocation stress, nursing home and organizational conditions, social support, addiction...etc.) (Raphael, 2004).

Once the environmental and behavioral factors are delineated and discussed between the advanced-practice gerontology nurse and other program members (e.g., other health care providers, managers), it is helpful to prioritize them in terms of importance (more important/ less important) and changeability (more susceptible to change / less susceptible to change) (Green \& Kreuter, 1991, p. 140). Objectives of smoking cessation / prevention programs must be formulated during this phase to achieve the outcomes of behavioral and environmental factors. Examples of these objectives were mentioned before (outcome and process objectives).

\subsection{Phase 4: Educational and Organizational Diagnosis: Factors Affecting Health Related Behavior and Environment}

This phase necessitates that the advanced practice gerontology nurse and other program team members identify predisposing, reinforcing, and enabling factors that increase the likelihood that behavioral and environmental changes will occur. In other words, predisposing, reinforcing, enabling factors that may affect smoking cessation/prevention program must be known before moving ahead in the program.

3.4.1 (a) The advanced-practice nurses and gerontology nurses assess the predisposing factors such as: knowledge, attitudes, beliefs, personal preferences, existing skills, and self-efficacy of older Canadians people in the nursing home who smoke as well as other staff nurses who are affected by their smoking. For example, advanced-practice gerontology nurses can use results from the literature to gain greater knowledge about the role of these factors in smoking. In addition, nurses also can ask the older Canadians people in the nursing home who smoke some focused questions to determine predisposing factors such as: Do you think you could stop smoking on your own? How would you describe your knowledge, skills, and selfconfidence to quit smoking? Please tell me about your attitudes and beliefs about smoking and smoking cessation.

3.4.2 (b) Reinforcing factors refer to those factors that reward or reinforce smoking cessation/prevention. For example, the contextual factors in nursing homes settings in Canada (e.g., organizational, social, psychological, economical, and cultural factors) may act as reinforcing factors.

3.4 .3 (c) Enabling factors guide the advanced-practice gerontology nurse to assess the motivation of older Canadians people in the nursing home who smoke, to engage themselves in smoking cessation and prevention programs. In addition, nurses assess the environmental enabling factors that may encourage or discourage smoking cessation/ prevention such as: (1) accessibility and availability of resources for smoking cessation, (2) availability of cigarettes both inside and/or geographically close to the nursing home, (3)"No Smoking" signs, and (4) the nature of laws and policies regarding smoking and smoking cessation issued by Canadian government, agencies, and the nursing home.

\subsection{Phase 5: Administrative and Policy Diagnosis: From Precede to Proceed}

The fifth phase aims to identify the policies, resources, and circumstances within the nursing home settings that may facilitate or hinder increased smoking cessation/ prevention programs. During this phase, advanced-practice gerontology nurses assess the availability of human and material resources and make plans to Proceed with the program. For example, nurses will likely wish to assess the time (date) frame for accomplishment of each objective using a Gantt chart; types and numbers of personnel (e.g., behavioral and addiction nurse specialist; smoking cessation consultant nurses; and related support staff ) needed to carry out the program; material resources and supplies (e.g., educational materials; booklets and pamphlets; printers; lecture room; data show projector; counseling clinic; medical and pharmacological supplies such as Nicotine/ Cotinine urine test kit; nicotine gum or patches ); and the organization budget.

The salient barriers for implementing the smoking cessation/prevention program must be identified during this phase. Examples of such barriers include: commitment, attitudes, program familiarity of those who will implement the program, complexity of the program, and adequate space for conducting the program. Moreover, policies, regulations, and organization in Canadian nursing homes must be examined for fitness with the health promotion program plan.

Upon completion of this fifth phase, the advancedpractice gerontology nurse and other team members involved with the health promotion program can turn their attention to implement the second part of the model, which is referred to as PROCEED and consists of four phases: six, seven, eight, and nine.

\subsection{Phase 6: Implementation}

Based on the policy and administrative diagnoses, the advanced-practice gerontology nurse can identify the interventions. Only two examples of these interventions will be discussed below. The first is an intervention to help older Canadian people who smoke in the nursing home to quit (smoking cessation counseling). The second is a preventive intervention to prevent second-hand smoking or to prevent a relapse among those who have already quit. 
3.6.1 (a) Smoking cessation counseling: This type of intervention represents intervention at an individual level. In this case, the advanced practice gerontology nurses assess the readiness of change among older Canadian people who smoke in the nursing home using Prochaska and DiClemente's (1983) Stages of Change Model. This model, which was developed by Prochaska and DiClemente over three decades, has become an influential theoretical model within the addiction behavior field. This model represents a motivational readiness continuum of changes toward healthy or unhealthy behaviors, and consists of six stages: pre-contemplation, contemplation, preparation, action, maintenance, and relapse (DiClemente, 2003; Prochaska \& DiClement, 1983).

Depending on the client's current stage of change, the nurse and client are able to decide which the most appropriate intervention for smoking cessation is. For example, they may select one or more of the following interventions: pharmacological (e.g., nicotine gum or patches), behavioral (e.g., group therapy), health education to increase client's level of knowledge, and social support.

3.6.2 (b) The preventive intervention: Advanced-practice gerontology nurses may provide health education using appropriate communication skills that foster teachinglearning for the other people and employees who do not smoke, or for those who already quit about smoking risks and cessation benefits. Examples of teaching-learning strategies that these nurses may use are: lectures, pamphlets, flayers, and intra-organizational e-mail networks or social media.

Having discussed the implementation phase of this hypothetical health promotion program, let us now turn to discuss the evaluation phases (7, 8, and 9).

\subsection{Process, Impact, and Outcome Evaluation (Phases 7, 8 , and 9)}

These final three phases of the PRECEDE-PROCEED model deal with process, impact, and outcome evaluation of the plan and intervention that were developed in earlier phases. First, a process evaluation should be conducted during the implementation of the smoking cessation/intervention program to ensure that the program was implemented according to plan. Second, an impact evaluation must be conducted to evaluate changes in predisposing, reinforcing, and enabling factors as well as changes in behavioral and environmental factors. Third, outcome evaluation measures the effect of the smoking cessation/ prevention program on health and quality of life for older Canadian people who smoke.

Advanced-practice gerontology nurses have a broad range of choices of evaluation models to evaluate the impact, process, and outcome of a smoking cessation/prevention program that is designed by advanced-practice gerontology nurses in Canadian nursing homes. One of the commonly used evaluation models is
"The logic Model for Planning Evaluation" (see McLaughlin \& Jordan, 1999).

\section{Conclusion}

The goal of building an evidence-based smoking cessation/prevention program for older Canadian people who smoke and live in nursing home by the advancedpractice gerontology nurses is commendable at both individual and organizational levels. First, this program is expected to help older Canadian people who smoke to quit, improve their health by decreasing the risk of diseases and mortality rates caused by smoking. Hence, this smoking cessation/prevention program will likely create a healthy nursing home environment that protects others from exposure to the hazards of second-hand smoke, and thereby facilitate disease prevention and enhance quality of life. Second, the implementation of a smoking prevention program will enhance maintenance for older Canadian people have quit smoking, and encourage those who have never smoked (e.g., other people and employees) to continue as free of smoke and negative smoking, this prevention program will likely provide up-to-date knowledge for these populations in regard to: the major risks of smoking; effective smoking cessation protocols; and the salient benefits of cessation. Subsequently, these nurses may use this knowledge to treat and prevent smoking among older Canadian people who smoke.

\section{References}

[1] British Columbia Ministry of Health. (2005). Healthy aging through healthy living: Towards a comprehensive policy and planning framework for Seniors in B.C. Retrieved May $1^{\text {st }}$, 2011 from http://www.health.gov.bc.ca/library/publications/year/2005/ healthy_aging.pdf

[2] Canadian Lung Association. (2008). the national lung health framework. Retrieved Feb.12, 2009, from http://www.lung.ca/about-propos/framework-cadre_e.php.

[3] Carosella, A. M., Ossip-Klein, D. J.,Watt, C. A., \& Podgorski, C. (2002). Smoking history, knowledge, and attitudes among older residents of a long-term care facility. Nicotine \& Tobacco Research, 4, 161-169.

[4] DiClemente, C. (2003). Addiction and change: How addictions develop and addicted people recover. New York: Guilford Press.

[5] Doolan, D. M., \& Froelicher, E. S. (2006). Efficacy of smoking cessation intervention among special populations: Review of the literature from 2000 to 2005. Nursing Research, 55, S29-S37.

[6] Edwards, P. \& Mawani, A. (2006). Healthy aging in Canada: A new vision, a vital investment from evidence to action - A background paper prepared for the Federal, Provincial and Territorial Committee of Officials (seniors). Ottawa, ON: Author. Retrieved May 5 ${ }^{\text {th }}, 2011$ from 
http://www.phac-aspc.gc.ca/seniorsaines/altformats/pdf/publications/public/healthysante/vision/vision-eng.pdf

[7] Gielen, A.C., \& McDonald, E.M. (1997). The PRECEDEPROCEED planning model. In: K., Glanz, F.M, Lewis, \& B.K. Rimmer (Eds.), Health Behavior and Education $\left(2^{\text {nd }}\right.$ Ed.) (pp. 359-383). San Francisco, CA: Jossey-Bass Publishers.

[8] Green, L.W., \& Kreuter, M. W. (2005). Health program planning: An educational and ecological approach ( $4^{\text {th }}$ Ed.). New York: McGraw-Hill.

[9] Green, L.W., \& Kreuter, M. W. (1991). Health promotion planning: An educational And environmental approach $\left(2^{\text {nd }}\right.$ Ed.). Mountain View, CA: Mayfield Publishers.

[10] Health Canada. (2002). Healthy aging - tobacco use and smoking cessation among seniors.

[11] Husten, C. G., Shelton, D. M., Chrismon, J. H., Lin, T. -C. W., Mowery, P., \& Powerll, F. A. (1997). Cigarette smoking and smoking cessation among older adults: United States, 1965-94. Tobacco Control, 6, 175-180.

[12] Kaplan, M. S., Newsom, J. T., \& McFarland, B. H. (2002). Older adults' contact with health practitioners: Is there an association with smoking practices? Journal of Gerontology. Biological Sciences \& Medical Sciences, 57(6), M343M346.

[13] La Croix, A. \& Omenn, G. (1992). Older adults and smoking. Clinics in Geriatric Medicine, 8(1), 69-87.
[14] McLaughlin, J. A., \& Jordan, G. B. (1999). Logic models: A tool for telling your program's performance story. Evaluation and Program Planning, 22, 65-72.

[15] Prochaska , J., \& Diclemente, C. (1983). Stages and processes of self-change of smoking: towards an integrated model of change. Journal of Consulting Clinical Psychology, 51, 390-395.

[16] Public Health Agency of Canada. (2009). [Analyses were performed using Health Canada's DAIS edition of anonymized microdata from the Canadian Tobacco Use Monitoring 2009, prepared by Health Canada].

[17] Raphael, D. (2004). Introduction to the social determinants of health. In D., Raphael (Ed.), Social determinants of health: Canadian perspectives (pp. 1-18). Toronto: Canadian Scholar's Press Inc.

[18] Statistics Canada. (2007). A portrait of seniors in Canada, 2006. (Ottawa: Statistics Canada). Retrieved May 1st, 2011 from http://www.statcan.gc.ca/pub/89-519-x/89-519x2006001-eng.pdf

[19] U.S. Department of Health and Human Services. (2004). The health consequences of smoking: $A$ report of the surgeon general. Retrieved from, http://www.ncbi.nlm.nih.gov/books/NBK44695/

[20] Watt, C., Carosella, A. M., Podgorski, C., \& Ossip-Klein, D. (2004). Attitudes toward giving smoking cessation advice among nursing staff at a long-term residential care facility. Psychology of Addictive Behaviors, 18 (1), 56-63. 